\title{
Methodology for the synthesis of 11,13-dihydroxy gibberellins
}

\author{
Jianping Liu, ${ }^{a}$ Lewis N. Mander, ${ }^{* a}$ and Masaji Koshioka ${ }^{b}$ \\ ${ }^{a}$ Research School of Chemistry, Institute of Advanced Studies, Australian National University, \\ Canberra ACT 0200, Australia. ${ }^{b}$ National Institute of Floricultural Science 2-1, Fujimoto, \\ Tsukuba, Ibaraki, Japan 305-8519 \\ E-mail:mander@rsc.anu.edu.au
}

\section{Dedicated to Professor Rod Rickards on the occasion of his $\mathbf{7 0}^{\text {th }}$ birthday}

(received 21 May 04; accepted 04 Jun 04; published on the web 11 Jun 04)

\begin{abstract}
A general procedure has been established for the synthesis of 11,13-dihydroxy gibberellins. The key steps involve the isomerization of the 19,10 lactone functionality to the 19,2-isomer, bromination at $\mathrm{C}-1$ and $\mathrm{C}-11$, then selective nucleophilic displacement of the 11-bromo substituent by acetate with silver(1) acetate. Reconstitution of the 19,10 lactone functionality was effected by bromolactonization of a $\Delta^{9}$-ene 19-carboxylic acid to give a $9 \beta$-bromo 19,10 -lactone followed by stereoselective hydrogenolysis of the bromo substituent.
\end{abstract}

Keywords: 11-Hydroxy-gibberellins, loquat, silver ion, nucleophilic substitution, bromolactonization

\section{Introduction}

Several gibberellins ("GAs") have been isolated from the seeds of immature fruit from loquat (Eriobotrya japonica ), ${ }^{1}$ including the 11-hydroxy derivatives $\mathrm{GA}_{35} \mathbf{1}, \mathrm{GA}_{80} 2$ and $\mathrm{GA}_{84} \mathbf{3}$, the structures of the latter two gibberellins being established by synthesis of the methyl ester 11 as outlined in Scheme 1. ${ }^{2}$
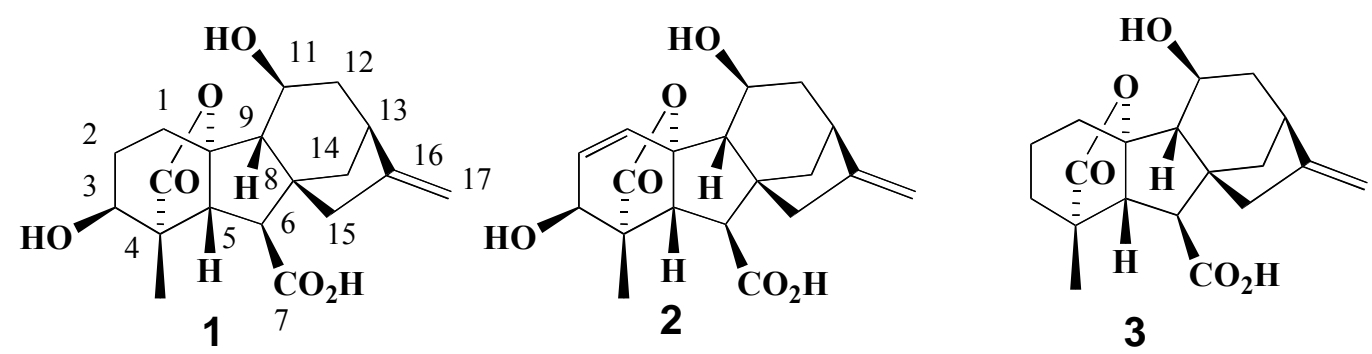


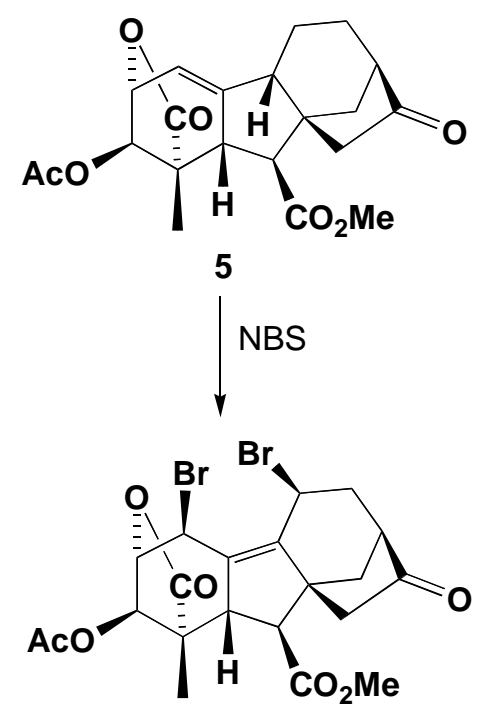

6

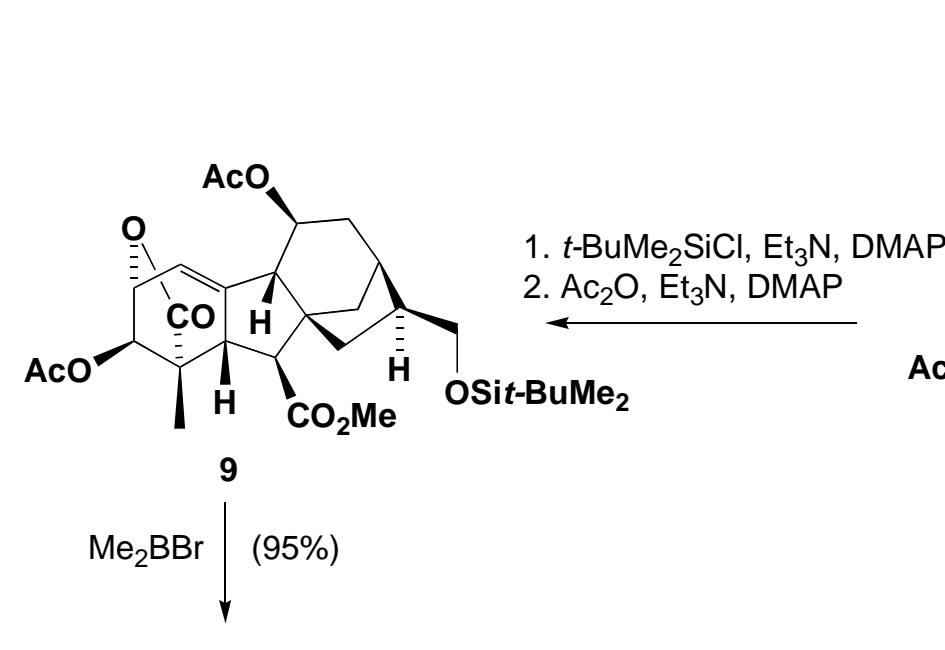

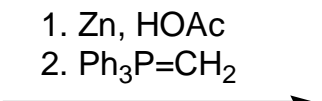

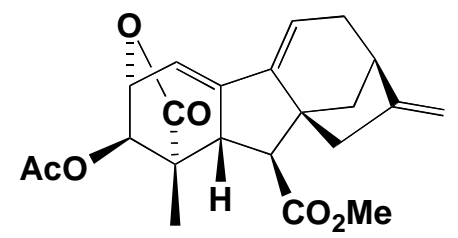

7

$\mathrm{BH}_{3} . \mathrm{SMe}_{2}$; $\mathrm{H}_{2} \mathrm{O}_{2}-\mathrm{NaOAc}$

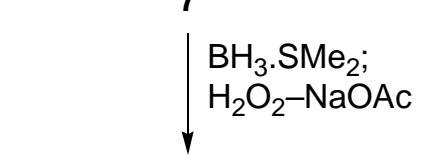

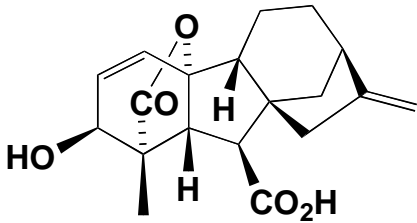

4

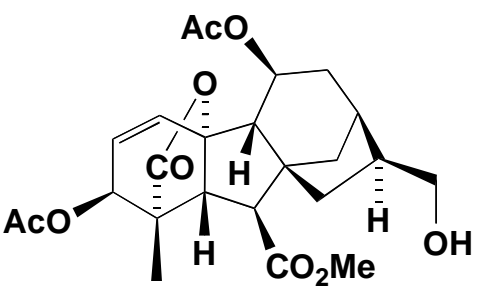

10

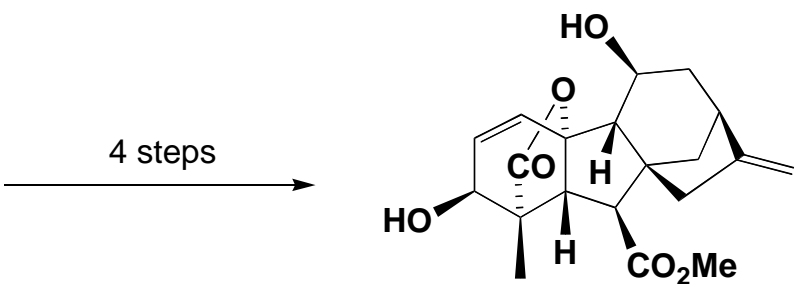

11

\section{Scheme 1}

The pivotal step in this sequence was the hydroboration of triene 7, whereby addition of borane to the hindered 9(11)-ene functionality is effected intramolecularly following hydroboration of the 16-ene bond. The mass spectra of some of the remaining unidentified GAs (measured on the trimethylsilylated methyl esters) from loquat showed a characteristic signature of a strong peak at m/z 295. 13-Hydroxy GAs typically give rise to a peak at $\mathrm{m} / \mathrm{z}$ 207/208 due to 
a C/D ring fragment and so we speculated that the unknown GAs might be hydroxylated at C-13 and possess an additional hydroxyl at C-11 (i.e. giving rise to a fragment with $\mathrm{m} / \mathrm{z} 207+88$ ), given that 12,13-, 13,14- and 13,15- substitution patterns could all be eliminated by comparison with reference compounds. ${ }^{3,4}$ We have therefore embarked upon the synthesis of GAs with the "missing" 11,13-dihydroxy pattern in order to provide a set of reference GAs. In this paper we disclose the successful synthesis of the methyl ester of $11 \alpha$-hydroxy gibberellin $A_{1}$ (13) from gibberellic acid $\left(\mathrm{GA}_{3}\right)(\mathbf{1 2})$.

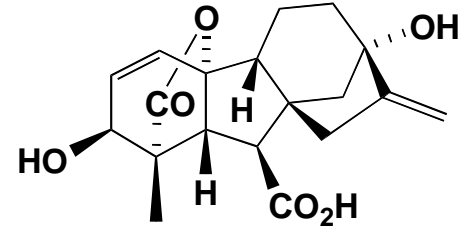

12

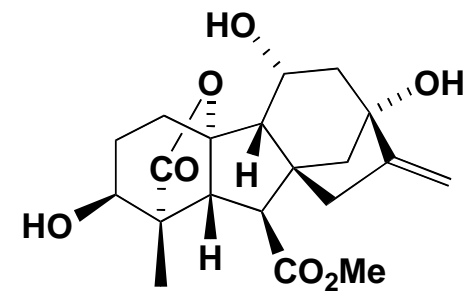

13

\section{Results and Discussion}

On first inspection it appeared that a simple duplication of the sequence outlined in Scheme 1, but starting with $\mathrm{GA}_{3}$ (12), would be effective. However, experience with the exploratory hydroboration of simple 13-hydroxylated GAs had been discouraging and so we elected to pursue a strategy based on the selective nucleophilic displacement of the allylic 11-bromo substituent on the 13-oxy analogue 16 of dibromide 6 (Scheme 2), expecting that the electronwithdrawing lactone function attached to C-2 would retard substitution of the 1-bromo substituent. To this end, ketone $\mathbf{1 4}{ }^{5}$ was prepared from 12 and treated with trifluoroacetic acid to afford the iso-lactone $\mathbf{1 5}$ which, when treated with $N$-bromosuccinimide and AIBN as the free radical initiator, was transformed into dibromide $16 .{ }^{6}$ When allowed to react with silver acetate in acetic acid, this product was converted into the triacetate $\mathbf{1 7}$ in a very satisfactory yield (83\%). Evidence for the 1,11-dibromo structure 16 came from C-1 and C-11 signals in ${ }^{13} \mathrm{C}$ NMR spectra at 33.4 and $33.8 \mathrm{ppm}$ as two methines with the corresponding protons at 4.80 and $5.13 \mathrm{ppm}$ respectively. Once substitution at C-11 was achieved, C-11 in $\mathbf{1 7}$ was shifted downfield from ca 33 ppm to $66.1 \mathrm{ppm}$, and H-11 to $5.52 \mathrm{ppm}$. H-12 $\alpha$ in 16 showed a coupling of $6.7 \mathrm{~Hz}$ to $\mathrm{H}-11$, but a larger value of $11.0 \mathrm{~Hz}$ in $\mathbf{1 7}$, consistent with the $11 \beta$ stereochemistry in the dibromide 16 and the $11 \alpha$ configuration in $\mathbf{1 7}$. Having introduced an oxy substituent at C-11, our next task was the restoration of the 19,2-lactone functionality. It appeared to be a reasonable proposition to attempt a reductive elimination of the 1-bromo substituent in $\mathbf{1 7}$, but on treatment with zinc metal in THF containing a few drops of acetic acid, diene 18 was obtained. When the reaction was conducted with a high concentration of acetic acid, however, the desired diene acid 19 was obtained. The ${ }^{1} \mathrm{H}$ NMR spectrum of this product indicated that three acetate groups had been 
retained ( $3 \mathrm{H}$ singlets at 2.07, 2.10 and $2.12 \mathrm{ppm}$ ) and the $\Delta^{1}$-alkene bond could be confirmed by the presence of a doublet at $6.22 \mathrm{ppm}$ for $\mathrm{H}-1$ and a doublet of doublets at $6.01 \mathrm{ppm}$ for $\mathrm{H}-2$.

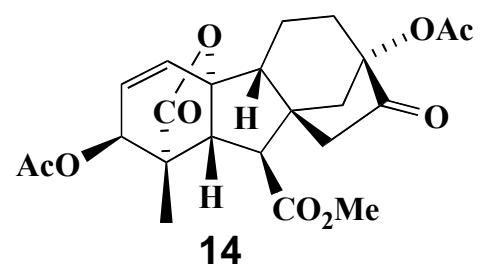

14

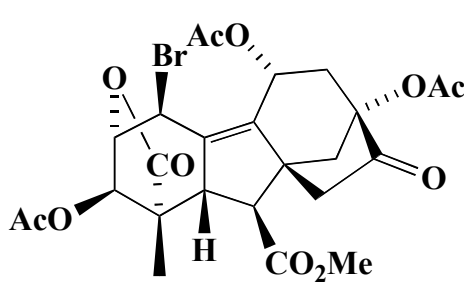

17

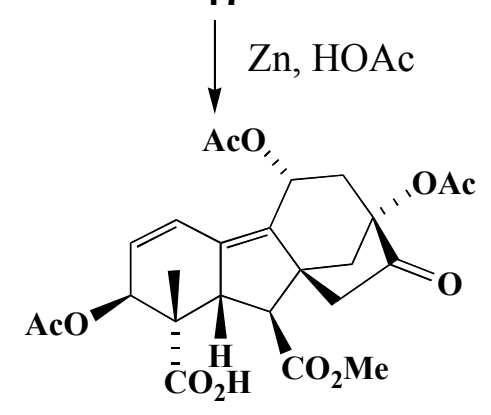

19
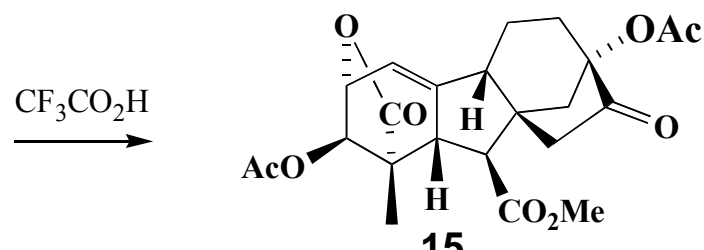

15
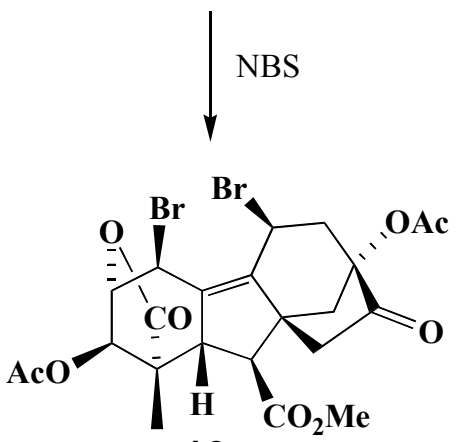

16

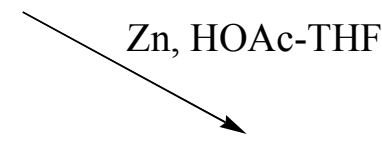

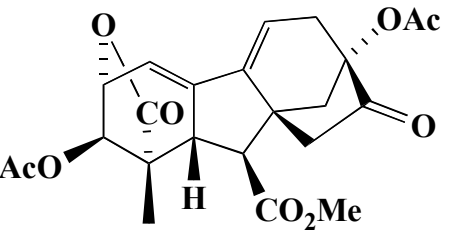

18

\section{Scheme 2}

With a view to reconstituting the A-ring lactone functionality (Scheme 3), selective hydrogenation of the $\Delta^{1}$ double bond in $\mathbf{1 9}$ was addressed, but proved to be problematical and we had to settle for a mixture of three reduction products: 9-ene $\mathbf{2 0}$ and the 1(10)-ene $\mathbf{2 1}$ as a mixture of 9-epimers. Separation proved to be difficult so the mixture was subjected to a bromo lactonization process, thereby affording lactone $\mathbf{2 2}$ in $68 \%$ overall yield accompanied by minor amounts of the lactone mixture 23. Separation was now feasible and removal of bromine from $\mathbf{2 2}$

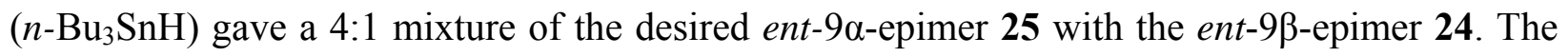
ent-9 $\alpha$-stereochemistry of $\mathbf{2 4}$ was apparent from the "normal" geminal coupling constant for H-5 and $\mathrm{H}-6$ of $10.0 \mathrm{~Hz}$, whereas the equivalent parameter for the minor ent-9ק-epimer was $12.2 \mathrm{~Hz}$ as observed for other ent-9ק-GAs. ${ }^{7}$ 


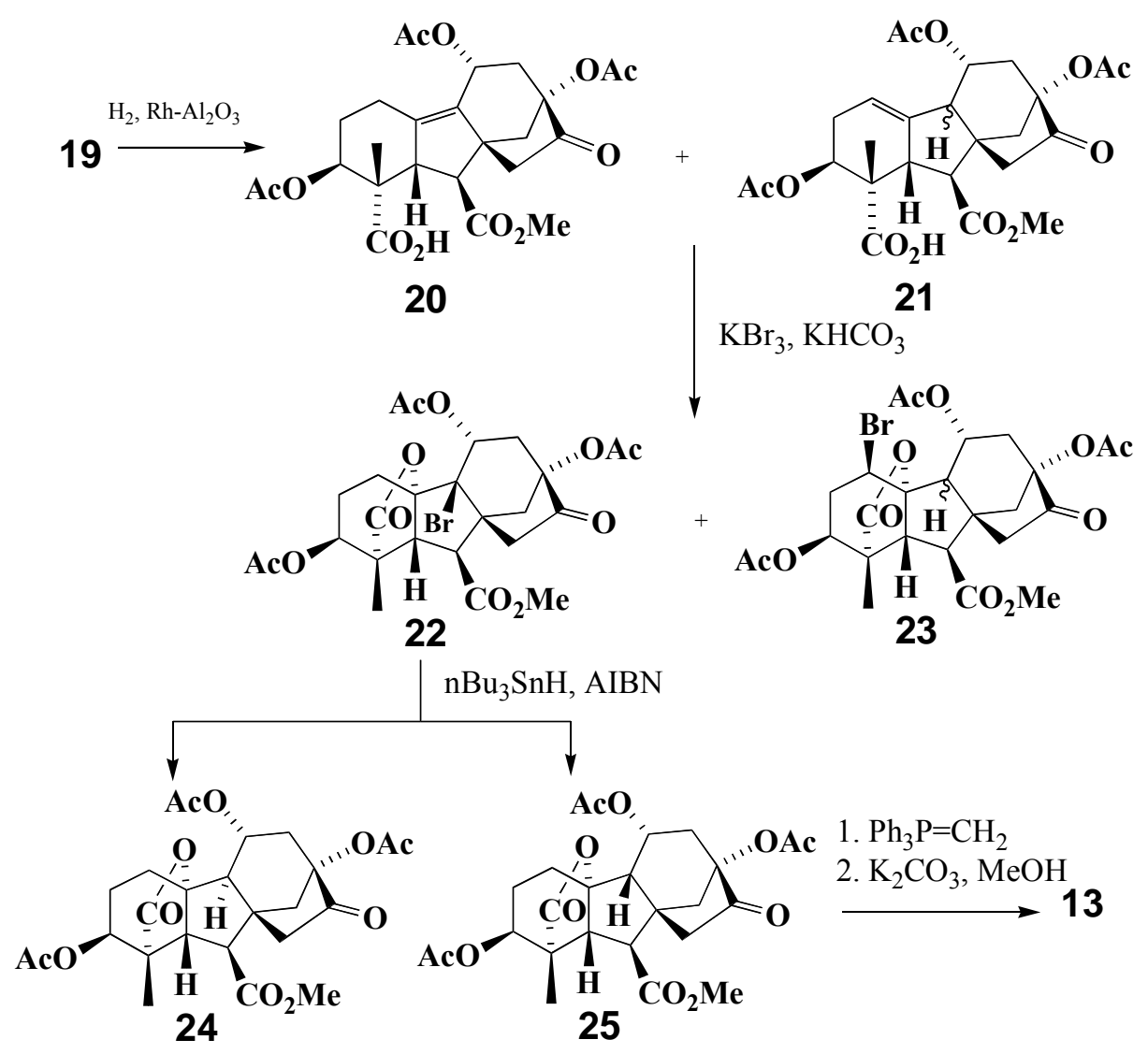

\section{Scheme 3}

With ketone $\mathbf{2 4}$ in hand, it was a simple task to complete the synthesis of $\mathbf{1 3}$ by means of a Wittig methylenation followed by hydrolysis. Under the conditions necessary to remove all acetate functions, epimerization at C-3 (due to the well-known retro-aldol process) was unavoidable $^{8}$ and a 1:2 mixture of $\mathbf{1 3}$ with its $3 \alpha$-epimer was obtained. We were pleased to find that the mass spectrum of the silylated derivative of $\mathbf{1 3}$ afforded a spectrum containing a strong peak at $\mathrm{m} / \mathrm{z} 295$, thereby providing strong support for our speculations regarding the structures of the unknown loquat GAs, although as yet no direct match between the synthetic and naturally occurring derivatives has been made.

\section{Experimental Section}

General Procedures. Infrared spectra $\left(v_{\max }\right)$ were recorded on a Perkin-Elmer 683 spectrophotometer in $0.25 \mathrm{~mm} \mathrm{NaCl}$ solution cells using deuterochloroform. NMR spectra were recorded on the following instruments: Varian Gemini 300 and Inova 500. For proton spectra recorded in chloroform, the residual peak of $\mathrm{CHCl}_{3}$ was used as the internal reference $(7.26 \mathrm{ppm})$ while the central peak of $\mathrm{CDCl}_{3}(77.0 \mathrm{ppm})$ was used as the reference for carbon spectra. Mass 
spectra $(70 \mathrm{eV})$ were recorded on a VG Micromass $7070 \mathrm{~F}$ double focussing mass spectrometer. Flash chromatography was conducted using Merck Kieselgel 60; medium pressure liquid chromatography (MPLC) was conducted using a Duramat ${ }^{\mathbb{B}}$ pump, a Waters Associates Differential Refractometer R40 diffractometer and Merck Lobar ${ }^{\circledR}$ Fertigsäule Größe LiChroprep ${ }^{\circledR}$ Si60 $(40-63 \mu \mathrm{m})$ columns.

ent-3 $\alpha, 13-D i a c e t o x y-2 \beta$-hydroxy-16-0xo-17,20-dinorgibberell-1(10)-ene-7,19-dioic acid 19,2lactone 7-methyl ester (15). Diacetate $14(9.0 \mathrm{~g}, 20 \mathrm{mmol})$ was treated with trifluoroacetic acid $(100 \mathrm{~mL})$ at room temperature for one hour and then dichloromethane $(100 \mathrm{~mL})$ was added to the solution. The reaction mixture was washed with brine and dried over sodium sulfate. The isolactone 15 (6.9 g, 77\% yield) was obtained as a colorless solid by chromatography on silica gel using ethyl acetate and hexane (1:3) as the eluting solvent and then crystallized from ethyl acetate and pentane, $\mathrm{mp} 185-186{ }^{\circ} \mathrm{C} ;{ }^{1} \mathrm{H}$ NMR $\delta 1.23(3 \mathrm{H}, \mathrm{s}, 4-\mathrm{Me}), 1.68\left(1 \mathrm{H}, \mathrm{dd}, J_{\mathrm{gem}}=11.2\right.$, $\left.J_{14 \alpha, 15 \beta}=3.3 \mathrm{~Hz}, \mathrm{H} 14 \alpha\right), 2.01(3 \mathrm{H}, \mathrm{s}, 13-\mathrm{OAc}), 2.12(3 \mathrm{H}, \mathrm{s}, 3-\mathrm{OAc}), 2.13\left(1 \mathrm{H}, \mathrm{dd}, J_{\mathrm{gem}}=17.2\right.$, $\left.J_{14 \alpha, 15 \beta}=3.3 \mathrm{~Hz}, \mathrm{H} 15 \beta\right), 2.39\left(1 \mathrm{H}\right.$, br d, $\left.J_{\text {gem }}=11.2 \mathrm{~Hz}, \mathrm{H} 14 \beta\right), 2.67\left(1 \mathrm{H}, \mathrm{d}, J_{5,6}=6.2 \mathrm{~Hz}, \mathrm{H} 6\right)$, $2.72(1 \mathrm{H}$, br d, $J=6.6 \mathrm{~Hz}, \mathrm{H} 9), 2.80\left(1 \mathrm{H}, \mathrm{d}, J_{\text {gem }}=17.2 \mathrm{~Hz}, \mathrm{H} 15 \alpha\right) 3.38\left(1 \mathrm{H}, \mathrm{dd}, J_{5,6}=6.2, J_{1,5}\right.$ $=2.5 \mathrm{~Hz}, \mathrm{H} 5), 3.75(3 \mathrm{H}, \mathrm{s}, \mathrm{OMe}), 5.04(2 \mathrm{H}, \mathrm{m}, \mathrm{H} 2, \mathrm{H} 3), 5.80(1 \mathrm{H}, \mathrm{m}, \mathrm{H} 1) .{ }^{13} \mathrm{C}$ NMR $\delta 17.1$ (C18), 17.1 (C11), 20.7, 20.8 (2×OAc), 31.6 (C12), 41.1, 44.9 (C14, C15), 45.2, 45.9 (C5, C6), 47.5, 47.6 (C4, C8), 48.8 (C9), 52.3 (OMe), 71.8, 75.3 (C2, C3), 80.3 (C13), 114.7 (C1), 150.6 (C10), 169.4, 170.2 (2×OAc), 175.1 (C7), 177.0 (C19), 211.0 (C16). IR ( $\left.v_{\max }\right)$ 3030, 1780, 1760, $1730 \mathrm{~cm}^{-1}$. LRMS (m/z) $446\left(\mathrm{M}^{+}, 7 \%\right), 430$ (3), 404 (4), 386 (25), 368 (15), 354 (43), 341 (18), 326 (13), 299 (26), 284 (15), 271 (52), 256 (38), 240 (30), 211 (100), 195 (33), 169 (43), 155 (65), 143 (28), 128 (14), 115 (13), 91 (17). HRMS found 446.1584; $\mathrm{C}_{23} \mathrm{H}_{26} \mathrm{O}_{9}$ requires 446.1577. Anal. found $\mathrm{C}, 61.62 ; \mathrm{H} 5.78 ; \mathrm{C}_{23} \mathrm{H}_{26} \mathrm{O}$ requires $\mathrm{C}, 61.88 ; \mathrm{H} 5.87$.

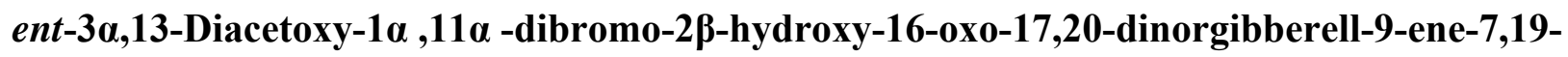
dioic acid 19,2-lactone 7-methyl ester (16). A solution of lactone 15 (6.7 g, $15 \mathrm{mmol})$, NBS $(5.9 \mathrm{~g}, 33 \mathrm{mmol})$ and AIBN $(80 \mathrm{mg})$ in carbon tetrachloride $(350 \mathrm{~mL})$ was heated at reflux for 20 min. The cooled mixture was filtered to remove solid (which was washed with $\mathrm{CCl}_{4}$ and the solutions combined). The mother liquor was then washed with brine and dried. The pure dibromide 16 was obtained $(6.4 \mathrm{~g}, 70 \%)$ by flash chromatography followed by MPLC separation (using ethyl acetate and hexane 1:2 as solvent in both cases), mp 125-126 ${ }^{\circ} \mathrm{C} .{ }^{1} \mathrm{H}$ NMR $\delta 1.14$ $(3 \mathrm{H}, \mathrm{s}, 4-\mathrm{Me}), 1.67\left(1 \mathrm{H}, \mathrm{dd}, J_{\mathrm{gem}}=11.1, J_{14 \alpha, 15 \beta}=3.2 \mathrm{~Hz}, \mathrm{H} 14 \alpha\right), 2.06(3 \mathrm{H}, \mathrm{s}, 13-\mathrm{OAc}), 2.20$ $(3 \mathrm{H}, \mathrm{s}, 3-\mathrm{OAc}), 2.35\left(1 \mathrm{H}, \mathrm{d}, J_{\mathrm{gem}}=17.6 \mathrm{~Hz}, \mathrm{H} 15 \alpha\right), 2.51\left(1 \mathrm{H}, \mathrm{dd}, J_{\mathrm{gem}}=15.0, J_{14 \alpha, 15 \beta}=2.5 \mathrm{~Hz}\right.$, $\mathrm{H} 12 \beta), 2.62\left(1 \mathrm{H}, \mathrm{dd}, J_{\mathrm{gem}}=15.0, J_{11,12 \alpha}=6.7 \mathrm{~Hz}, \mathrm{H} 12 \alpha\right), 2.78\left(1 \mathrm{H}, \mathrm{dd}, J_{\mathrm{gem}}=17.6, J_{14 \alpha, 15 \beta}=3.2\right.$ $\mathrm{Hz}, \mathrm{H} 15 \beta), 3.08\left(1 \mathrm{H}, \mathrm{d}, J_{5,6}=8.8 \mathrm{~Hz}, \mathrm{H} 6\right), 3.18\left(1 \mathrm{H}, \mathrm{dd}, J_{\mathrm{gem}}=11.1, J_{12 \beta, 14 \beta}=2.5 \mathrm{~Hz}, \mathrm{H} 14 \beta\right)$, $3.80(3 \mathrm{H}, \mathrm{s}, \mathrm{OMe}), 4.07\left(1 \mathrm{H}, \mathrm{d}, J_{5,6}=8.8 \mathrm{~Hz}, \mathrm{H} 5\right), 4.80\left(1 \mathrm{H}, \mathrm{d}, J_{1,2}=3.3 \mathrm{~Hz}, \mathrm{H1}\right), 5.03(1 \mathrm{H}, \mathrm{dd}$, $\left.J_{2,3}=4.7, J_{1,2}=3.3 \mathrm{~Hz}, \mathrm{H} 2\right), 5.06\left(1 \mathrm{H}, \mathrm{d}, J_{2,3}=4.7 \mathrm{~Hz}, \mathrm{H} 3\right), 5.13\left(1 \mathrm{H}, \mathrm{d}, J_{11,12 \alpha}=6.7 \mathrm{~Hz}, \mathrm{H} 11\right)$. ${ }^{13} \mathrm{C}$ NMR $\delta 14.9$ (C18), 20.7, 20.9 (2×OAc), 33.4, 33.8 (C1, C11), 43.4 (C12), 44.4, 44.6 (C14, C15), 46.3 (C6), 46.6 (C8), 49.3 (C5), 52.1 (C4), 52.4 (OMe), 73.1, 73.4 (C2, C3), 82.2 (C13), 130.9 (C9), 145.9 (C10), 168.8, 170.1 (2×OAc), 171.3 (C7), 173.4 (C19), 207.5 (C16). IR ( $\left.v_{\max }\right)$ 
3030, 1790, 1765, $1735 \mathrm{~cm}^{-1}$. LRMS (m/z) $606\left(\mathrm{M}^{+}, 1 \%\right), 604\left(\mathrm{M}^{+}, 1.2\right), 602\left(\mathrm{M}^{+}, 0.8\right), 575$ (1.5), 573 (2.8), 571 (1.4), 538 (2), 536 (3.8), 534 (2), 525 (40), 523 (40), 483 (68), 481 (68), 444 (20), 423 (12), 401 (10), 379 (10), 315 (8), 297 (34), 280 (46), 254 (60), 237 (43), 221 (28), 211 (41), 195 (100), 181 (38), 167 (46), 153 (26), 141 (20), 128 (20), 115 (20), 80 (12). HRMS (M ${ }^{+}$ $\mathrm{Br}$ ) found 523.0593; $\mathrm{C}_{23} \mathrm{H}_{24} \mathrm{O} 9 \mathrm{Br}$ requires 523.0603.

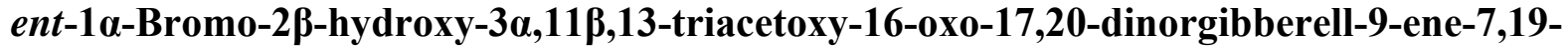
dioic acid 19,2-lactone 7-methyl ester (17). The dibromide 16 (5.0 g, $8.3 \mathrm{mmol})$ in acetic acid $(150 \mathrm{~mL})$ was treated with silver acetate $(2.5 \mathrm{~g}, 15 \mathrm{mmol})$ at $50-55^{\circ} \mathrm{C}$ overnight. The solution was diluted with ethyl acetate $(150 \mathrm{~mL})$, neutralized with cold $\mathrm{NaHCO}_{3}$ (aq.), then the organic layer washed with brine and dried. Flash column separation with ethyl acetate and hexane (1:3$1: 2)$ as eluting solvent gave triacetate $17(4.0 \mathrm{~g}, 83 \%)$ as a colorless solid, which could be further purified by recrystallization from ethyl acetate and pentane, mp 206-208 ${ }^{\circ} \mathrm{C} .{ }^{1} \mathrm{H}$ NMR $\delta 1.15$ $(3 \mathrm{H}, \mathrm{s}, 4-\mathrm{Me}), 1.82\left(1 \mathrm{H}, \mathrm{dd}, J_{\text {gem }}=11.2, J_{14 \alpha, 15 \beta}=2.4 \mathrm{~Hz}, \mathrm{H} 14 \alpha\right), 1.92\left(1 \mathrm{H}, \mathrm{dd}, J_{\text {gem }}=11.1\right.$, $\left.J_{11,12 \alpha}=11.0 \mathrm{~Hz}, \mathrm{H} 12 \alpha\right), 2.05$ (3H, s, 13-OAc), 2.15 (3H, s, 11-OAc), 2.20 (3H, s, 3-OAc), 2.50 $\left(1 \mathrm{H}, \mathrm{ddd}, J_{\mathrm{gem}}=11.1, J_{11,12 \beta}=7.8, J_{12 \beta, 14 \beta}=2.7 \mathrm{~Hz}, \mathrm{H} 12 \beta\right), 3.08\left(1 \mathrm{H}, \mathrm{d}, J_{5,6}=9.6 \mathrm{~Hz}, \mathrm{H} 6\right), 3.12$ $\left(1 \mathrm{H}, \mathrm{dd}, J_{\mathrm{gem}}=11.2, J_{12 \beta, 14 \beta}=2.7 \mathrm{~Hz}, \mathrm{H} 14 \beta\right), 3.79(3 \mathrm{H}, \mathrm{s}, \mathrm{OMe}), 4.01\left(1 \mathrm{H}, \mathrm{dd}, J_{5,6}=9.6, J_{5,11}=\right.$ $4.5 \mathrm{~Hz}, \mathrm{H} 5), 5.00\left(1 \mathrm{H}, \mathrm{dd}, J_{2,3}=5.0, J_{1,2}=2.3 \mathrm{~Hz}, \mathrm{H} 2\right), 5.07\left(1 \mathrm{H}, \mathrm{d}, J_{2,3}=5.0 \mathrm{~Hz}, \mathrm{H} 3\right), 5.31(1 \mathrm{H}$, $\left.\mathrm{d}, J_{1,2}=2.3 \mathrm{~Hz}, \mathrm{H} 1\right), 5.52\left(1 \mathrm{H}, \mathrm{ddd}, J_{11,12 \alpha}=11.0, J_{11,12 \beta}=7.8, J_{5,11}=4.5 \mathrm{~Hz}, \mathrm{H} 11\right) .{ }^{13} \mathrm{C} \mathrm{NMR} \delta$ 15.5 (C18), 20.7, 20.8, 21.2 (3×OAc), 34.7 (C1), 39.8 (C12), 43.6, 44.4 (C14, C15), 46.1 (C8), 46.6, 49.8 (C5, C6), 52.5 (C4), 52.7 (OMe), 66.1 (C11), 73.5, 73.9 (C2, C3), 82.0 (C13), 128.5 (C9), 142.8 (C10), 168.8, 169.3, 170.4 (3×OAc), 171.7 (C7), 173.8 (C19), 207.6 (C16). IR (v $\max )$ 3040, 1790, 1760, $1735 \mathrm{~cm}^{-1}$. LRMS ( $\left.\mathrm{m} / \mathrm{z}\right) 583\left(\mathrm{M}^{+}-1,1 \%\right), 581\left(\mathrm{M}^{+}-1,1\right), 553(1), 551(1), 524$ (10), 522 (10), 503 (41), 482 (8), 480 (8), 461 (28), 444 (12), 422 (21), 420 (23), 401 (23), 383 (10), 357 (28), 341 (30), 313 (22), 297 (32), 280 (34), 269 (41), 254 (50), 237 (58), 211 (78), 195 (100), 181 (56), 169 (50), 155 (55), 141 (30), 128 (29), 115 (23), 105 (12), 91 (22), 77 (12), 61 (30). HRMS $\left(\mathrm{M}^{+}-1\right)$ found 583.0634, 581.0648; $\mathrm{C}_{25} \mathrm{H}_{26} \mathrm{O}_{11} \mathrm{Br}$ requires 583.0638, 581.0658. Anal. found C, 51.64; H, 4.62; Br, 13.44; $\mathrm{C}_{25} \mathrm{H}_{27} \mathrm{O}_{11} \mathrm{Br}$ requires $\mathrm{C}, 51.47 ; \mathrm{H}, 4.66 ; \mathrm{Br}, 13.70$.

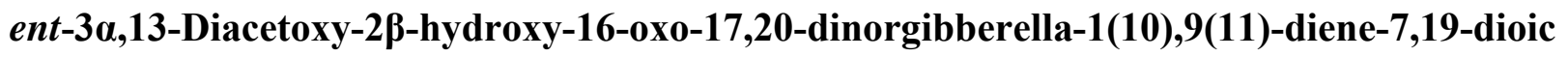
acid 19,2-lactone 7-methyl ester (18). A solution of the triacetate 17 (18 $\mathrm{mg}, 0.03 \mathrm{mmol})$ in THF $(20 \mathrm{~mL})$ containing a few drops of acetic acid was treated with zinc dust (100 $\mathrm{mg}$, washed with $1 \mathrm{M} \mathrm{HCl}$, water, methanol, ether and dried under high vacuum before use) and stirred at room temp overnight. The zinc was removed by filtration through Celite ${ }^{\circledR}$ and the solid was washed with ethyl acetate $(30 \mathrm{~mL})$. The solvent was removed under reduced pressure, ethyl acetate $(6 \mathrm{~mL})$ was added, and the solution was washed with brine and dried. The diene 18 was then obtained (10 mg, 75\%) by flash chromatography with ethyl acetate and hexane $(1: 2-1: 1)$ as eluting solvent. ${ }^{1} \mathrm{H}$ NMR $\delta 1.24(3 \mathrm{H}, \mathrm{s}, 4-\mathrm{Me}), 1.89\left(1 \mathrm{H}, \mathrm{dd}, J_{\text {gem }}=10.4, J_{14 \alpha, 15 \beta}=2.7 \mathrm{~Hz}\right.$, $\mathrm{H} 14 \alpha), 2.07$ (3H, s, 13-OAc), $2.12(3 \mathrm{H}, \mathrm{s}, 3-\mathrm{OAc}), 2.37\left(1 \mathrm{H}, \mathrm{d}, J_{\text {gem }}=17.0 \mathrm{~Hz}, \mathrm{H} 15 \alpha\right), 2.45(1 \mathrm{H}$, $\left.\mathrm{dd}, J_{\mathrm{gem}}=18.7, J_{11,12}=4.4 \mathrm{~Hz}, \mathrm{H} 12\right), 2.48\left(1 \mathrm{H}, \mathrm{dd}, J_{\mathrm{gem}}=17.0, J_{14 \alpha, 15 \beta}=2.7 \mathrm{~Hz}, \mathrm{H} 15 \beta\right), 2.66$ $\left(1 \mathrm{H}, \mathrm{d}, J_{5,6}=12.3 \mathrm{~Hz}, \mathrm{H} 6\right), 2.69\left(1 \mathrm{H}, \mathrm{dd}, J_{\mathrm{gem}}=18.7, J_{11,12^{\prime}}=4.3 \mathrm{~Hz}, \mathrm{H}^{\prime} 12\right), 3.02\left(1 \mathrm{H}, \mathrm{d}, J_{\mathrm{gem}}=\right.$ $10.4 \mathrm{~Hz}, \mathrm{H} 14 \beta), 3.42\left(1 \mathrm{H}, \mathrm{dd}, J_{5,6}=12.3, J_{1,5}=3.2 \mathrm{~Hz}, \mathrm{H} 5\right), 3.78(3 \mathrm{H}, \mathrm{s}, \mathrm{OMe}), 4.99\left(1 \mathrm{H}, \mathrm{d}, J_{2,3}\right.$ 
$=4.8 \mathrm{~Hz}, \mathrm{H} 3), 5.01\left(1 \mathrm{H}\right.$, dd, overlap with H3, H2), $5.95\left(1 \mathrm{H}, \mathrm{t}, J_{11,12}=4.0 \mathrm{~Hz}, \mathrm{H} 11\right), 6.16(1 \mathrm{H}$, br t, $J=4.0 \mathrm{~Hz}, \mathrm{H} 1) .{ }^{13} \mathrm{C} \mathrm{NMR} \delta 18.1$ (C18), 20.7, 20.8 (2×OAc), 36.6 (C12), 39.9 (C14), 45.7 (C6), 47.4 (C15), 47.5, 48.1 (C4, C8), 49.4 (C5), 52.5 (OMe), 71.8, 75.1 (C2, C3), 83.1 (C13), 115.9, 116.9 (C1, C11), 142.6, 143.5 (C9, C10), 169.6, 170.1 (2×OAc), 171.8 (C7), 174.4 (C19), $209.6(\mathrm{C} 16)$. IR $\left(v_{\max }\right) 3060,1765,1740,1640 \mathrm{~cm}^{-1}$. LRMS $(\mathrm{m} / \mathrm{z}) 444\left(\mathrm{M}^{+}, 23 \%\right), 384(6), 360$ (23), 339 (8), 297 (41), 280 (75), 254 (66), 211 (28), 195 (100), 167 (22), 115 (14), 91 (11), 77 (7). HRMS found 444.1419; $\mathrm{C}_{23} \mathrm{H}_{24} \mathrm{O}_{9}$ requires 444.1420 .

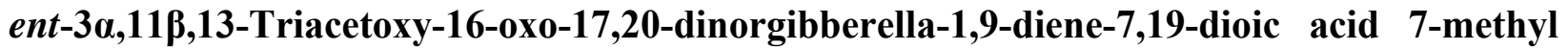
ester (19). The bromide 17 (268 mg, $0.46 \mathrm{mmol})$ and zinc powder (1.0 g, washed with $1 \mathrm{M} \mathrm{HCl}$, water, methanol, ether and dried under high vacuum before use) in acetic acid (15 mL) and THF $(5 \mathrm{~mL})$ was stirred at room temperature overnight. The zinc was removed by filtration through Celite $^{\circledR}$ and the solid was washed with ethyl acetate $(30 \mathrm{~mL})$. The desired acid (181) (135 mg, $58 \%)$ by chromatography with ethyl acetate and hexane as eluting solvents $(1: 2-2: 3)$ then using ethyl acetate, hexane and acetic acid (2:3:0.1), when the lactone was eluted off. Diene acid 19 was recrystallized from ethyl acetate and pentane, mp $150-152{ }^{\circ} \mathrm{C} .{ }^{1} \mathrm{H}$ NMR $\delta 1.23(3 \mathrm{H}, \mathrm{s}, 4-$ $\mathrm{Me}), 1.96\left(1 \mathrm{H}, \mathrm{dd}, J_{\mathrm{gem}}=11.1, J_{11,12 \alpha}=10.9 \mathrm{~Hz}, \mathrm{H} 12 \alpha\right), 2.072 .10,2.12(3 \times 3 \mathrm{H}, \mathrm{s}, 3 \times \mathrm{OAc}), 2.20$ $\left(1 \mathrm{H}, \mathrm{dd}, J_{\mathrm{gem}}=17.6, J_{14 \alpha, 15 \beta}=2.9 \mathrm{~Hz}, \mathrm{H} 15 \beta\right), 2.39\left(1 \mathrm{H}, \mathrm{d}, J_{\mathrm{gem}}=17.6 \mathrm{~Hz}, \mathrm{H} 15 \alpha\right), 2.47(1 \mathrm{H}$, ddd, $\left.J_{\text {gem }}=11.1, J_{11,12 \beta}=7.7, J_{12 \beta, 14 \beta}=2.5 \mathrm{~Hz}, \mathrm{H} 12 \beta\right), 3.10\left(1 \mathrm{H}, \mathrm{dd}, J_{\text {gem }}=10.9, J_{12 \beta, 14 \beta}=2.5 \mathrm{~Hz}\right.$, $\mathrm{H} 14 \beta), 3.75$ (3H, s, OMe), 3.75 (2H, m, overlap with OMe, H5, H6), 5.59 (1H, d, J2,3 = 5.6 Hz, H3), $5.59\left(1 \mathrm{H}, \mathrm{m}\right.$, overlap with H3, H11), $6.01\left(1 \mathrm{H}, \mathrm{dd}, J_{1,2}=9.8, J_{2,3}=5.6 \mathrm{~Hz}, \mathrm{H} 2\right), 6.62(1 \mathrm{H}, \mathrm{d}$, $\left.J_{1,2}=9.8 \mathrm{~Hz}, \mathrm{H} 1\right) .{ }^{13} \mathrm{C}$ NMR $\delta 19.6$ (C18), 20.9, 21.0, $21.0(3 \times \mathrm{OAc}), 40.3$ (C12), 44.7, 44.8 (C14, C15), 47.9 (C8), 48.7, 49.5 (C5, C6), 52.3 (OMe), 53.7 (C4), 66.8 (C11), 70.0 (C3), 82.6 (C13), 126.3, 127.7 (C1, C2), 129.9 (C9), 134.7 (C10), 169.3, 169.3, 170.3 (3×OAc), 173.5 (C7), 177.6 (C19), 208.8 (C16). IR ( $\left.v_{\max }\right) 3500,3020,3010,1760,1730 \mathrm{~cm}^{-1}$. LRMS (m/z) $504\left(\mathrm{M}^{+}\right.$, 0.4\%), $503\left(\mathrm{M}^{+}-1,1.3\right), 460$ (4), 444 (50), 418 (6), 402 (10), 384 (30), 339 (29), 297 (50), 280 (75), 254 (73), 237 (53), 221 (45), 195 (100), 181 (41), 167 (45), 153 (31), 141 (20), 128 (19), 115 (18), 91 (8). HRMS $\left(\mathrm{M}^{+}-1\right)$ found 503.1557; $\mathrm{C}_{25} \mathrm{H}_{27} \mathrm{O}_{11}$ requires 503.1553. Anal. foundC, $59.21 ; \mathrm{H}, 5.59 ; \mathrm{C}_{25} \mathrm{H}_{28} \mathrm{O}_{11}$ requires $\mathrm{C}, 59.52 ; \mathrm{H}, 5.59$.

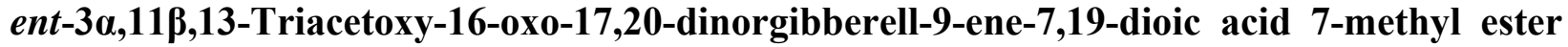

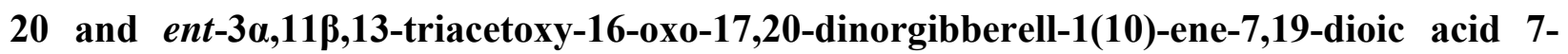
methyl ester (21). A solution of diene $19(100 \mathrm{mg}, 0.2 \mathrm{mmol})$ and rhodium on alumina (5\%, $10 \mathrm{mg})$ in THF $(100 \mathrm{~mL})$ was stirred under a hydrogen atmosphere at room temperature overnight. The catalyst was removed by filtration through Celite ${ }^{\circledR}$ and the product $(100 \mathrm{mg})$ was obtained without further purification, although NMR showed it was a mixture of $\mathbf{2 0}$ and the two C-9 epimers of the isomeric alkene 21.

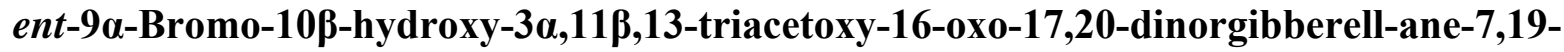

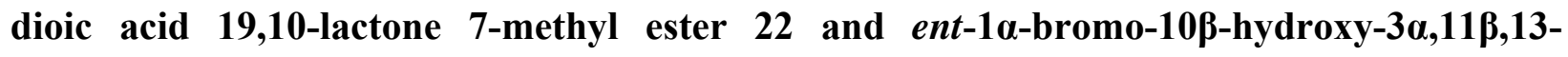
triacetoxy-16-0xo-17,20-dinorgibberellane-7,19-dioic acid 19,10-lactone 7-methyl ester (23). A mixture of alkenes 20 and 21 (100 mg, $0.2 \mathrm{mmol})$ dissolved in THF (15 mL) and potassium bicarbonate (1.0 M aq., $6 \mathrm{~mL}$ ) was treated with potassium tribromide $(1.0 \mathrm{M}$ aq., $5 \mathrm{~mL}$, prepared 
by the addition of bromine to potassium bromide solution at room temperature and stirring for 1 h) at $0{ }^{\circ} \mathrm{C}$ for $1 \mathrm{~h}$. Potassium bisulfite solution $(15 \% \mathrm{aq}, 10 \mathrm{~mL})$ was added, followed by ethyl acetate $(20 \mathrm{~mL})$. The organic phase was washed with brine, dried and the products $23(10 \mathrm{mg})$ (from 21) followed by 22 (79 $\mathrm{mg}, 68 \%$ ) (from 20) were obtained by chromatography with ethyl acetate and hexane $(1: 3-1: 1)$ as eluting solvents.

Data for 22: ${ }^{1} \mathrm{H}$ NMR $\delta 1.16(3 \mathrm{H}, \mathrm{s}, 4-\mathrm{Me}), 2.052 .12,2.16(3 \times 3 \mathrm{H}, \mathrm{s}, 3 \times \mathrm{OAc}), 2.36(1 \mathrm{H}$, ddd, $\left.J_{\text {gem }}=11.4, J_{11,12 \beta}=7.4, J_{12 \beta, 14 \beta}=3.0 \mathrm{~Hz}, \mathrm{H} 12 \beta\right), 2.63\left(1 \mathrm{H}, \mathrm{d}, J_{5,6}=6.4 \mathrm{~Hz}, \mathrm{H} 6\right), 2.77(1 \mathrm{H}, \mathrm{dd}$, $\left.J_{\text {gem }}=11.4, J_{14 \alpha, 15 \beta}=3.8 \mathrm{~Hz}, \mathrm{H} 14 \alpha\right), 2.95\left(1 \mathrm{H}, \mathrm{dd}, J_{\mathrm{gem}}=11.4, J_{12 \beta, 14 \beta}=3.0 \mathrm{~Hz}, \mathrm{H} 14 \beta\right), 3.11$ $\left(1 \mathrm{H}, \mathrm{dd}, J_{\text {gem }}=17.9, J_{14 \alpha, 15 \beta}=3.8 \mathrm{~Hz}, \mathrm{H} 15 \beta\right), 3.26\left(1 \mathrm{H}, \mathrm{d}, J_{\text {gem }}=17.9 \mathrm{~Hz}, \mathrm{H} 15 \alpha\right), 3.77(3 \mathrm{H}, \mathrm{s}$, OMe), $3.84\left(1 \mathrm{H}, \mathrm{d}, J_{5,6}=6.4 \mathrm{~Hz}, \mathrm{H} 5\right), 5.04\left(1 \mathrm{H}, \mathrm{dd}, J_{2,3}=3.2,2.5 \mathrm{~Hz}, \mathrm{H} 3\right), 5.61\left(1 \mathrm{H}, \mathrm{dd}, J_{11,12 \alpha}\right.$ $\left.=11.2, J_{11,12 \beta}=7.4 \mathrm{~Hz}, \mathrm{H} 11\right) .{ }^{13} \mathrm{C}$ NMR $\delta 14.4(\mathrm{C} 18), 20.6,20.7,21.1$ (3×OAc), 26.7, $29.1(\mathrm{C} 1$, C2), 39.8 (C12), 44.5, 45.1 (C14, C15), 48.5 (C8), 49.8 (C6), 52.3 (OMe), 52.7 (C5), 55.5 (C4), 71.3 (C3), 75.6 (C11), 80.3 (C9), 80.9 (C13), 95.1 (C10), 168.6, 169.5, 170.1 (3×OAc), 170.8 (C7), 176.5 (C19), 207.9 (C16). IR ( $\left.v_{\max }\right)$ 1765, $1740 \mathrm{~cm}^{-1}$. LRMS (m/z) 586, $584\left(\mathrm{M}^{+}, 14 \%\right)$, 558 (18), 556 (18), 543 (38), 541 (37), 505 (18), 484 (41), 482 (40), 463 (20), 438 (28), 436 (29), 403 (81), 385 (13), 375 (60), 329 (39), 315 (100), 297 (56), 283 (76), 269 (82), 255 (98), 237 (49), 227 (54), 211 (66), 197 (44), 181 (32), 169 (49), 153 (14), 143 (25), 129 (22), 115 (14), 91 (18). HRMS found 586.0877, 584.0893; $\mathrm{C}_{25} \mathrm{H}_{29} \mathrm{O}_{11} \mathrm{Br}$ requires 586.0873, 584.0893.

Data for 23: ${ }^{1} \mathrm{H}$ NMR $\delta 1.10(3 \mathrm{H}, \mathrm{s}, 4-\mathrm{Me}), 2.05,2.10,2.13(3 \times 3 \mathrm{H}, \mathrm{s}, 3 \times \mathrm{OAc}), 2.59(1 \mathrm{H}, \mathrm{dd}$, $\left.J_{\text {gem }}=11.3, J_{14 \alpha, 15 \beta}=2.6 \mathrm{~Hz}, \mathrm{H} 14 \alpha\right), 2.76\left(1 \mathrm{H}, \mathrm{d}, J_{5,6}=10.1 \mathrm{~Hz}, \mathrm{H} 6\right), 2.83\left(1 \mathrm{H}, \mathrm{d}, J_{\mathrm{gem}}=11.3\right.$ $\mathrm{Hz}, \mathrm{H} 14 \beta), 3.75(3 \mathrm{H}, \mathrm{s}, \mathrm{OMe}), 3.84\left(1 \mathrm{H}, \mathrm{d}, J_{5,6}=10.1 \mathrm{~Hz}, \mathrm{H} 5\right), 4.57\left(1 \mathrm{H}, \mathrm{dd}, J_{1,2}=3.4,3.1 \mathrm{~Hz}\right.$, $\mathrm{H} 1), 4.86\left(1 \mathrm{H}, \mathrm{dd}, J_{2,3}=2.8,2.7 \mathrm{~Hz}, \mathrm{H} 3\right), 5.53\left(1 \mathrm{H}, \mathrm{dd}, J_{11,12 \alpha}=9.7, J_{11,12 \beta}=5.8 \mathrm{~Hz}, \mathrm{H} 11\right) .{ }^{13} \mathrm{C}$ NMR $\delta 14.3$ (C18), 20.8, 21.1, 21.5 (3×OAc), 33.8 (C2), 38.0 (C12), 39.5 (C15), 43.3 (C1), 46.8 (C14), 47.6 (C8), 48.3 (C6), 50.9 (C5), 51.5 (C4), 51.5 (C9), 52.6 (OMe), 63.9 (C11), 70.4 (C3), 81.8 (C13), 93.1 (C10), 169.7, 170.1, 170.3 (3×OAc), 171.2 (C7), 175.1 (C19), 210.1 (C16). IR $\left(v_{\max }\right) 1770,1735 \mathrm{~cm}^{-1}$. LRMS (m/z) 587, $585\left(\mathrm{M}^{+}+1,1 \%\right), 558$ (11), 556 (11), 543 (19), 541 (18), 516 (8), 514 (8), 498 (8), 496 (8), 485 (16), 483 (17), 456 (32), 454 (31), 435 (18), 417 (32), 393 (8), 375 (28), 357 (38), 343 (25), 315 (70), 299 (22), 283 (33), 271 (68), 255 (40), 239 (26), 227 (28), 211 (100), 197 (30), 183 (38), 169 (62), 155 (36), 143 (32), 129 (22), 115 (15), 105 (16), 91 (22). HRMS $\left(\mathrm{M}^{+}+1\right)$ found 587.0971, 585.0966; $\mathrm{C}_{25} \mathrm{H}_{30} \mathrm{O}_{11} \mathrm{Br}$ requires 587.0951, 585.0971 .

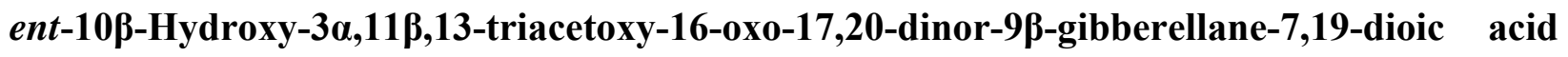

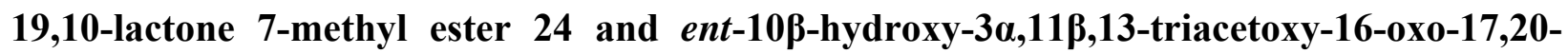
dinorgibberellane-7,19-dioic acid 19,10-lactone 7-methyl ester (25). The bromide 22 (40 mg, $0.068 \mathrm{mmol})$, AIBN (4 mg) and tributyltin hydride $(0.04 \mathrm{~mL})$ in benzene $(15 \mathrm{~mL})$ was heated under reflux for $40 \mathrm{~min}$. The mixture was diluted with ethyl acetate $(10 \mathrm{~mL})$, washed with brine and dried. The ent-9 $\beta$-isomer 24 (4.5 mg, 13\%) followed by 25 (22.0 mg, 64\%) were obtained by chromatography on silica gel with ethyl acetate and hexane $(1: 3-1: 1)$ as eluting solvents.

Data for 25: mp 250-252 ${ }^{\circ} \mathrm{C} .{ }^{1} \mathrm{H}$ NMR $\delta 1.07$ (3H, s, 4-Me), 2.06, 2.12, $2.13(3 \times 3 \mathrm{H}, \mathrm{s}, 3 \times \mathrm{OAc})$, $2.38\left(1 \mathrm{H}, \mathrm{d}, J_{\mathrm{gem}}=17.1 \mathrm{~Hz}, \mathrm{H} 15 \alpha\right), 2.63\left(1 \mathrm{H}, \mathrm{dd}, J_{\text {gem }}=11.2, J_{14 \alpha, 15 \beta}=3.1 \mathrm{~Hz}, \mathrm{H} 14 \alpha\right), 2.78(1 \mathrm{H}$, 
$\left.\mathrm{d}, J_{5,6}=10.0 \mathrm{~Hz}, \mathrm{H} 6\right), 2.82\left(1 \mathrm{H}, \mathrm{d}, J_{\mathrm{gem}}=11.2 \mathrm{~Hz}, \mathrm{H} 14 \beta\right), 3.16\left(1 \mathrm{H}, \mathrm{d}, J_{5,6}=10.0 \mathrm{~Hz}, \mathrm{H} 5\right), 3.75$ $(3 \mathrm{H}, \mathrm{s}, \mathrm{OMe}), 4.96\left(1 \mathrm{H}, \mathrm{dd}, J_{2,3}=3.3,2.5 \mathrm{~Hz}, \mathrm{H} 3\right), 5.56\left(1 \mathrm{H}, \mathrm{dd}, J_{11,12 \beta}=10.4, J_{9,11}=6.0 \mathrm{~Hz}\right.$, H11). ${ }^{13} \mathrm{C}$ NMR $\delta 14.4$ (C18), 20.8, 21.1, 21.6 (3×OAc), 25.3, 28.2 (C1, C2), 38.5 (C12), 39.6 (C15), 47.4 (C14), 48.2 (C8), 51.1 (C6), 51.7 (C4), 52.6 (OMe), 53.1 (C5), 55.4 (C9), 64.4 (C11), 71.0 (C3), 81.9 (C13), 91.2 (C10), 169.7, 170.1, 170.3 (3×OAc), 171.9 (C7), 176.0 (C19), $210.4(\mathrm{C} 16)$. IR $\left(v_{\max }\right) 1785,1765,1735 \mathrm{~cm}^{-1}$. LRMS $(\mathrm{m} / \mathrm{z}) 506\left(\mathrm{M}^{+}, 1 \%\right), 478(46), 463(18)$, 436 (33), 418 (100), 376 (98), 358 (62), 316 (78), 288 (43), 270 (96), 217 (63), 169 (38), 143 (35), 91 (26). HRMS found 506.1785; $\mathrm{C}_{25} \mathrm{H}_{30} \mathrm{O}_{11}$ requires 506.1788.

Data for 24: ${ }^{1} \mathrm{H}$ NMR $\delta 1.04(3 \mathrm{H}, \mathrm{s}, 4-\mathrm{Me}), 2.05,2.06,2.15(3 \times 3 \mathrm{H}, \mathrm{s}, 3 \times \mathrm{OAc}), 2.24(1 \mathrm{H}, \mathrm{dd}$, $\left.J_{\text {gem }}=18.8, J_{14 \alpha, 15 \beta}=3.1 \mathrm{~Hz}, \mathrm{H} 15 \beta\right), 2.39\left(1 \mathrm{H}, \mathrm{d}, J_{\mathrm{gem}}=18.8 \mathrm{~Hz}, \mathrm{H} 15 \alpha\right), 2.62\left(1 \mathrm{H}, \mathrm{d}, J_{9,11}=11.7\right.$ $\mathrm{Hz}, \mathrm{H} 9), 2.77\left(1 \mathrm{H}, \mathrm{d}, J_{5,6}=12.2 \mathrm{~Hz}, \mathrm{H} 6\right), 2.99\left(1 \mathrm{H}, \mathrm{dd}, J_{\mathrm{gem}}=11.2, J_{12 \beta, 14 \beta}=2.3 \mathrm{~Hz}, \mathrm{H} 14 \beta\right), 3.10$ $\left(1 \mathrm{H}, \mathrm{d}, J_{5,6}=12.2 \mathrm{~Hz}, \mathrm{H} 5\right), 3.74(3 \mathrm{H}, \mathrm{s}, \mathrm{OMe}), 4.89\left(1 \mathrm{H}, \mathrm{ddd}, J_{9,11}=11.7, J_{11,12 \alpha}=10.3, J_{11,12 \beta}=\right.$ $5.9 \mathrm{~Hz}, \mathrm{H} 11), 4.99\left(1 \mathrm{H}, \mathrm{dd}, J_{2,3}=3.4,2.5 \mathrm{~Hz}, \mathrm{H} 3\right) .{ }^{13} \mathrm{C} \mathrm{NMR} \delta 14.0(\mathrm{C} 18), 20.8,20.9,21.1$ (3×OAc), 25.9, 27.8 (C1, C2), 38.8 (C12), 40.8, 41.5 (C14, C15), 48.8 (C8), 48.9 (C6), 52.1 (C4), 52.5 (OMe), 53.7 (C5), 58.7 (C9), 65.5 (C11), 71.2 (C3), 83.5 (C13), 89.2 (C10), 169.1, 169.7, $170.0(3 \times \mathrm{OAc}), 170.2$ (C7), 175.5 (C19), 205.7 (C16). IR ( $\left.v_{\max }\right)$ 1780, 1760, $1735 \mathrm{~cm}^{-}$ ${ }^{1} . \mathrm{LRMS}(\mathrm{m} / \mathrm{z}) 506\left(\mathrm{M}^{+}, 2 \%\right), 476(8), 464(28), 446$ (30), 428 (8), 418 (16), 404 (100), 386 (30), 376 (20), 358 (22), 342 (34), 326 (10), 316 (28), 300 (29), 282 (38), 270 (32), 256 (38), 242 (28), 229 (18), 211 (36), 197 (30), 185 (20), 169 (28), 159 (16), 143 (22), 129 (20), 107 (8), 91 (12), 79 (10). HRMS found 506.1791; $\mathrm{C}_{25} \mathrm{H}_{30} \mathrm{O}_{11}$ requires 506.1788.

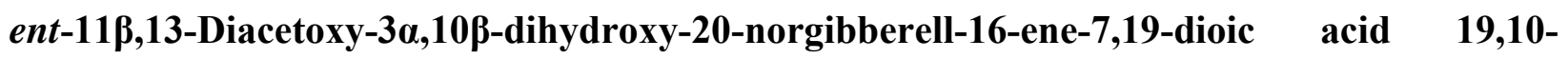

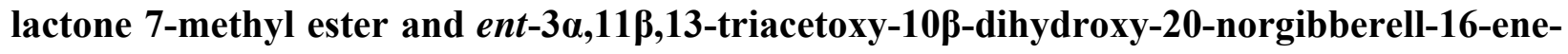
7,19-dioic acid 19,10-lactone 7-methyl ester. A clear yellow ylide solution was prepared by stirring a mixture of $\left[\mathrm{Ph}_{3} \mathrm{PCH}_{3}\right]^{+} \mathrm{Br}^{-}(90 \mathrm{mg})$ and $n$-BuLi $(2.5 \mathrm{M}$ in hexane, $0.09 \mathrm{~mL})$ in benzene $(5 \mathrm{~mL})$ at room temperature. Pentane $(5 \mathrm{~mL})$ was added after $30 \mathrm{~min}$., and the supernatant cannulated into the ketone $25(15.3 \mathrm{mg}, 0.03 \mathrm{mmol})$ at room temperature. The solution was kept stirring overnight and the reaction was stopped by adding $\mathrm{NH}_{4} \mathrm{Cl}$ solution $(0.5 \mathrm{~mL})$ to the reaction mixture at room temperature. The organic layer was diluted with ethyl acetate $(5 \mathrm{~mL})$, washed with brine, dried and the solvent removed. The title triacetate $(1 \mathrm{mg}, 7 \%)$, followed by the title 11,13 -diacetate $(3.5 \mathrm{mg}, 25 \%)$ was obtained after chromatographic separation with ethyl acetate and pentane $(1: 3-1: 2)$ as eluting solvent.

Data for diacetate: ${ }^{1} \mathrm{H}$ NMR $\delta 1.16(3 \mathrm{H}, \mathrm{s}, 4-\mathrm{Me}), 2.01,2.08(2 \times 3 \mathrm{H}, \mathrm{s}, 2 \times \mathrm{OAc}), 2.70\left(1 \mathrm{H}, \mathrm{d}, J_{5,6}\right.$ $=8.7 \mathrm{~Hz}, \mathrm{H} 6), 3.18\left(1 \mathrm{H}, \mathrm{d}, J_{5,6}=8.7 \mathrm{~Hz}, \mathrm{H} 5\right), 3.71(3 \mathrm{H}, \mathrm{s}, \mathrm{OMe}), 3.83(1 \mathrm{H}, \mathrm{br} \mathrm{s}, \mathrm{H} 3), 5.03(1 \mathrm{H}$, s, H17), $5.08\left(1 \mathrm{H}, \mathrm{d}, J=1.7 \mathrm{~Hz}, \mathrm{H}^{\prime} 17\right), 5.46\left(1 \mathrm{H}, \mathrm{dd}, J_{11,12 \beta}=13.7, J_{9,11}=6.9 \mathrm{~Hz}, \mathrm{H} 11\right) .{ }^{13} \mathrm{C}$ NMR $\delta 14.5$ (C18), 21.5, 22.0 (2×OAc), 28.1, 28.3 (C1, C2), 42.0 (C12), 42.5, 44.3 (C14, C15), 50.0 (C6), 50.9 (C8), 52.2 (OMe), 52.8 (C5), 52.9 (C4), 53.7 (C9), 66.8 (C11), 70.2 (C3), 83.1 (C13), 92.3 (C10), 108.1 (C17), 150.1 (C16), 169.4, 170.5 (2×OAc), 173.1 (C7), 177.8 (C19). LRMS (m/z) $462\left(\mathrm{M}^{+}, 2 \%\right), 446$ (2), 431 (5), 420 (30), 402 (10), 371 (15), 360 (70), 342 (36), 332 (58), 314 (36), 298 (100), 282 (50), 254 (55), 239 (60), 221 (36), 211 (76), 195 (52), 183 
(38), 169 (39), 155 (46), 143 (40), 129 (50), 115 (32), 105 (56), 91 (77), 77 (44). HRMS found $462.1891 ; \mathrm{C}_{24} \mathrm{H}_{30} \mathrm{O}_{9}$ requires 462.1890 .

Data for triacetate: ${ }^{1} \mathrm{H}$ NMR $\delta 1.07(3 \mathrm{H}, \mathrm{s}, 4-\mathrm{Me}), 2.02,2.092 .11(3 \times 3 \mathrm{H}, \mathrm{s}, 3 \times \mathrm{OAc}), 2.70(1 \mathrm{H}$, $\left.\mathrm{d}, J_{5,6}=9.0 \mathrm{~Hz}, \mathrm{H} 6\right), 3.12\left(1 \mathrm{H}, \mathrm{d}, J_{5,6}=9.0 \mathrm{~Hz}, \mathrm{H} 5\right), 3.73(3 \mathrm{H}, \mathrm{s}, \mathrm{OMe}), 4.95\left(1 \mathrm{H}, \mathrm{t}, J_{2,3}=2.6\right.$ $\mathrm{Hz}, \mathrm{H} 3), 5.04(1 \mathrm{H}, \mathrm{s}, \mathrm{H} 17), 5.10\left(1 \mathrm{H}, \mathrm{d}, J=1.8 \mathrm{~Hz}, \mathrm{H}^{\prime} 17\right), 5.48\left(1 \mathrm{H}, \mathrm{dd}, J_{11,12 \beta}=13.3, J_{9,11}=6.7\right.$ Hz, H11). LRMS (m/z) $504\left(\mathrm{M}^{+}, 2 \%\right), 473$ (1), 462 (38), 444 (10), 420 (15), 413 (24), 402 (98), 384 (26), 374 (50), 360 (29), 340 (60), 324 (20), 314 (50), 298 (56), 270 (100), 255 (50), 239 (52), 221 (80), 211 (60), 195 (49), 179 (30), 164 (34), 155 (38), 141 (30), 129 (38), 115 (22), 107 (18), 91 (46), 79 (25). HRMS found 504.1997; $\mathrm{C}_{26} \mathrm{H}_{32} \mathrm{O}_{10}$ requires 504.1995.

ent-3 $\alpha, 10 \beta, 11 \beta, 13-T e t r a h y d r o x y-20$-norgibberell-16-ene-7,19-dioic acid 19,10-lactone 7-

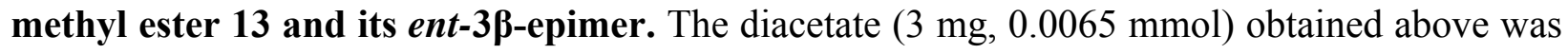
treated with potassium carbonate $(0.3 \mathrm{mg})$ in methanol at room temperature for $5 \mathrm{~h}$. The solvent was evaporated under reduced pressure and triol $13(0.6 \mathrm{mg}, 27 \%)$, followed by the ent-3 $\beta$ epimer ( $1.3 \mathrm{mg}, 54 \%)$ were obtained after separation by chromatography on silica gel with ethyl acetate and pentane $(2: 1-1: 0)$ as eluting solvent.

Data for 13: ${ }^{1} \mathrm{H}$ NMR $\delta 1.16(3 \mathrm{H}, \mathrm{s}, 4-\mathrm{Me}), 2.71\left(1 \mathrm{H}, \mathrm{dd}, J_{\mathrm{gem}}=10.3, J_{14 \alpha, 15 \beta}=2.3 \mathrm{~Hz}, \mathrm{H} 14 \alpha\right)$, $2.77\left(1 \mathrm{H}, \mathrm{d}, J_{5,6}=10.6 \mathrm{~Hz}, \mathrm{H} 6\right), 3.28\left(1 \mathrm{H}, \mathrm{d}, J_{5,6}=10.6 \mathrm{~Hz}, \mathrm{H} 5\right), 3.72(3 \mathrm{H}, \mathrm{s}, \mathrm{OMe}), 3.85(1 \mathrm{H}$, br s, H3), 4.39 (1H, m, H11), $4.98(1 \mathrm{H}, \mathrm{s}, \mathrm{H} 17), 5.28(1 \mathrm{H}, \mathrm{dd}, J=2.6,2.1 \mathrm{~Hz}, \mathrm{H} 17) .{ }^{13} \mathrm{C}$ NMR $\delta$ 14.5 (C18), 28.0, 28.6 (C1, C2), 43.5 (C12), 45.3, 49.1 (C14, C15), 49.5 (C8), 50.5 (C6), 51.9 (C5), 52.1 (OMe), 52.8 (C4), 56.4 (C9), 66.1 (C11), 70.2 (C3), 77.7 (C13), 94.1 (C10), 108.3 (C17), 156.0 (C16), 172.5 (C7), 176.5 (C19). IR ( $\left.v_{\max }\right)$ 3450, 3050, 1760, 1735, $1660 \mathrm{~cm}^{-1}$. LRMS (m/z) $378\left(\mathrm{M}^{+}, 13 \%\right), 360$ (13), 346 (22), 328 (36), 318 (43), 300 (50), 282 (34), 270 (70), 255 (50), 239 (41), 211 (66), 195 (46), 185 (30), 167 (66), 155 (36), 143 (45), 135 (68), 129 (50), 115 (46), 105 (58), 91 (100), 85 (32), 77 (70). HRMS found 378.1680; $\mathrm{C}_{20} \mathrm{H}_{26} \mathrm{O}_{7}$ requires 378.1679. LRMS of tris-trimethylsilyl ether (m/z) $594\left(\mathrm{M}^{+}, 19 \%\right), 489$ (9), $562(9), 504$ (20), 462 (8), 445 (8), 375 (13), 311 (8), 295 (38), 239 (40), 191 (10), 117 (15), 73 (100).

Data for the ent-3ß-epimer: ${ }^{1} \mathrm{H}$ NMR $\delta 1.19(3 \mathrm{H}, \mathrm{s}, 4-\mathrm{Me}), 2.55\left(1 \mathrm{H}, \mathrm{d}, J_{5,6}=9.7 \mathrm{~Hz}, \mathrm{H} 5\right), 2.65$ $\left(1 \mathrm{H}, \mathrm{dd}, J_{\text {gem. }}=10.5, J_{14 \alpha, 15 \beta}=2.1 \mathrm{~Hz}, \mathrm{H} 14 \alpha\right), 2.81\left(1 \mathrm{H}, \mathrm{d}, J_{5,6}=9.7 \mathrm{~Hz}, \mathrm{H} 6\right), 3.70(1 \mathrm{H}$, overlap with OMe, H3), $3.71(3 \mathrm{H}, \mathrm{s}, \mathrm{OMe}), 4.33(1 \mathrm{H}, \mathrm{m}, \mathrm{H} 11), 4.97(1 \mathrm{H}, \mathrm{s}, \mathrm{H} 17), 5.25(1 \mathrm{H}, \mathrm{dd}, J=2.5$, $\left.2.3 \mathrm{~Hz}, \mathrm{H}^{\prime} 17\right) .{ }^{13} \mathrm{C}$ NMR $\delta 12.7$ (C18), 29.4, 31.5 (C1, C2), 43.2 (C12), 45.9, 49.0 (C14, C15), 50.4 (C6), 50.7 (C8), 52.2 (OMe), 52.7 (C4), 55.8 (C5), 57.7 (C9), 66.2 (C11), 73.1 (C3), 77.5 (C13), 92.7 (C10), 108.3 (C17), 155.4 (C16), 172.6 (C7), 176.1 (C19). IR (v $\left.v_{\max }\right)$ 3450, 3050, 1765, 1735, $1665 \mathrm{~cm}^{-1}$. LRMS (m/z) $378\left(\mathrm{M}^{+}, 23 \%\right), 360$ (27), 342 (32), 328 (66), 314 (40), 300 (68), 282 (53), 255 (55), 239 (45), 213 (52), 195 (60), 167 (55), 149 (43), 135 (49), 117 (33), 105 (63), 91 (100), 77 (59). HRMS found 378.1683; $\mathrm{C}_{20} \mathrm{H}_{26} \mathrm{O}_{7}$ requires 378.1679. 


\section{References}

1. (a) Yuda, E.; Yokota, T.; Nonaka, M.; Fukushima, Y.; Nakagawa, S.; Katsura, N.; Ota, Y.; Murofushi, N.; Takahashi, N. Chemistry Express 1987, 2, 499. (b) Koshioka, M.; Pearce, D.; Pharis R. P.; Murakami, Y. Agric. Biol. Chem. 1988, 52, 1353. (c) Yuda E.; Nakagawa S.; Murofushi N.; Takahashi N.; Yokota T.; Koshioka M.; Murakami Y.; Pearce D.; Pharis R. P.; Kraft-Klaunzer P.; Mander L. N.; Patrick G. L. Biosci. Biotech. Biochem. 1992, 56, 17.

2. (a) Mander, L. N.; Patrick, G. L. Tetrahedron Lett. 1990, 31, 423. (b) Mander L.N.; KraftKlaunzer P. Phytochemistry 1992, 31, 2519.

3. Gaskin, P.; MacMillan, J. GC-MS of Gibberellins and Related Compounds: Methodology and a Library of Reference Spectra; Cantocks Enterprises: Bristol, 1991.

4. Liu, J.; Mander L. N.; Willis, A. C. Tetrahedron 1998, 54, 11,637.

5. Lombardo L., Mander L. N.; Turner J. V. Aust. J. Chem. 1981, 34, 745.

6. Cf. Furber, M.; Mander, L. N.; Patrick, G. L. J. Org. Chem. 1990, 55, 4860.

7. Furber, M.; Kraft-Klaunzer, P.; Mander, L. N.; Pour, M.; Yamauchi, T.; Murofushi, N.; Yamane, H.; Schraudolf, H. Aust. J. Chem. 1995, 48, 427.

8. MacMillan, J.; Pryce, R. J. J. Chem. Soc. 1967, 740. 